\title{
Status of Phytophthora and Huanglongbing Diseases of Citrus Causing Decline in Coorg Mandarin in India
}

Priti Sonavane and Venkataravanappa Venkataravanappa

Division of Plant Pathology, Central Horticultural Experiment Station, Indian Council of Agriculture Research, Indian Institute of Horticultural Research, Hessaraghatta Lake PO, Chettalli, Bangalore 571248, India

\begin{abstract}
Coorg mandarin is a famous ecotype of mandarin grown in multi-tier cropping system in coffee and pepper plantations in Coorg region of India. The roving survey indicated disease incidence of Huanglongbing (HLB) and Phytophthora, ranging between $35 \%-64.2 \%$ and $22.5 \%-35.6 \%$ in different places of Coorg region. A total of 177 Phytophthora infected (plant roots 59, soil 118), and 576 greening or HLB infected Coorg mandarin samples were collected from 184 Coorg mandarin orchards, of these 111 Phytophthora isolates were isolated and characterized in this study on the basis of colony growth patterns and morphological structures. Based on the virulence, only 52 of 111 Phytophthora isolates were amplified by PCR using universal internal transcriber spacer (ITS) primers, cloned and sequenced. The sequences analysis of Phytophthora isolates revealed more than $97 \%$ nucleotide (nt) similarity within themselves, except eight isolates of $P$. palmivora and four isolates of $P$. nicotianae had varied nt identity ( $70.6 \%$ to 97.4\%) with other Phytophthora species compared. Similarly, 523 of 576 Coorg mandarin samples were confirmed the presence of HLB infection by PCR using 16S rRNA gene specific universal primers. The representative five of 523 Coorg mandarin isolates showed nt identity ranged from $94.4 \%$ to $95.3 \%$ with Candidatus Liberibacter asiaticus (AB008366) isolate infecting citrus in Asia and one isolate of HLB shared nt identity of $96.9 \%$ with Ca. L. asiaticus (KJ944269).
\end{abstract}

Key words: Phytophthora, citrus greening, gummosis, RPARH medium, Asian psyllid.

\section{Introduction}

Citrus is considered as one of the most important tropical fruit crop in India. It plays a vital role in the fruit economy of the country, next to mango and banana. India ranks sixth among top citrus producing countries of the world. In India, citrus is primarily grown in Assam, Andhra Pradesh, Maharashtra, Punjab, Karnataka, Uttaranchal, Bihar, Orissa and Gujarat. Andhra Pradesh occupies first place in citrus production by producing 1,805.64 tonnes, constituting $24.19 \%$ share of total production in India [1].

The citrus cultivation in India is hampered due to non-availability of disease-free planting material, bud wood transmissible diseases, general neglect, scarcity of water and poor management practices [2]. Beside this, Citrus spp. are prone to be attacked by

Corresponding author: Priti Sonavane, Ph.D., research field: plant pathology. more than 150 diseases and disorders caused by fungal, bacterial, viral and few non-viral pathogens right from nursery level to harvesting stage resulting in considerable yield losses [3]. The most important commercial citrus in India is the mandarin orange, followed by the sweet oranges and acid limes. Coorg mandarin is a famous ecotype of mandarin grown in Kodagu, Hassan and Chikmagalur of districts of Karnataka (India) as a component of the multi-tier cropping system with coffee and pepper plantations for more than 150 years with an area of 24,000 ha. Coorg oranges are regarded as man-made hybrids of mandarins (Citrus reticulata). Greenish-yellow in colour, they have a tight skin and a sweet-sour taste, unlike Nagpur oranges, which are known to have loose skin and sweet taste. Coorg oranges are said to have longer shelf life compared to other varieties [4]. This cropping system proved efficient with higher land use efficiency. The excess shade and sub 
optimal management practices affect the growth and fruiting of the trees.

In recent years, the Coorg mandarin cultivation area has come down to less than 2,000 ha. The region was, in fact, known as an orange belt till the Phytophthora rot and citrus greening disease took a heavy toil of crop [5]. The yield of single plant has come down to around $10 \mathrm{~kg}$, which was once more than $50 \mathrm{~kg}$. Diseases related to Phytophthora and greening or Huanglongbing (HLB) disease are difficult to estimate as both cause decline of trees [6-8]. The most important Phytophthora sp. reported to cause diseases in Coorg mandarin include $P$. palmivora, P. parasitica Dastur (P. nicotianae) and P. citrophthora [9]. These Phytophthora species have been shown to cause some serious soil borne diseases of citrus, including damping off of seedlings in the seedbed, root and crown rot in nurseries, foot rot and brown rot of fruits. The second important disease, which severely hampered the production of Coorg mandarin, is citrus greening disease. Candidatus Liberibacter asiaticus is the most predominant species in the region, which is vectored by Asian psyllid (Diaphorina citri Kuwayama) [10]. Differences in aggressiveness, competitiveness and response to fungicides varied among these two pathogens. Therefore, the aim of the present study was to characterize the Phytophthora and HLB disease pathogens associated with Coorg mandarin decline and also to know the status of disease on Coorg mandarin.

\section{Materials and Methods}

2.1 Survey, Collection of Disease Samples and Disease Assessment

The roving survey was employed during 2015-2016 in 184 different farmers' fields in Kodagu and Hassan districts (Karnataka) in India (Fig. 1), to estimate the

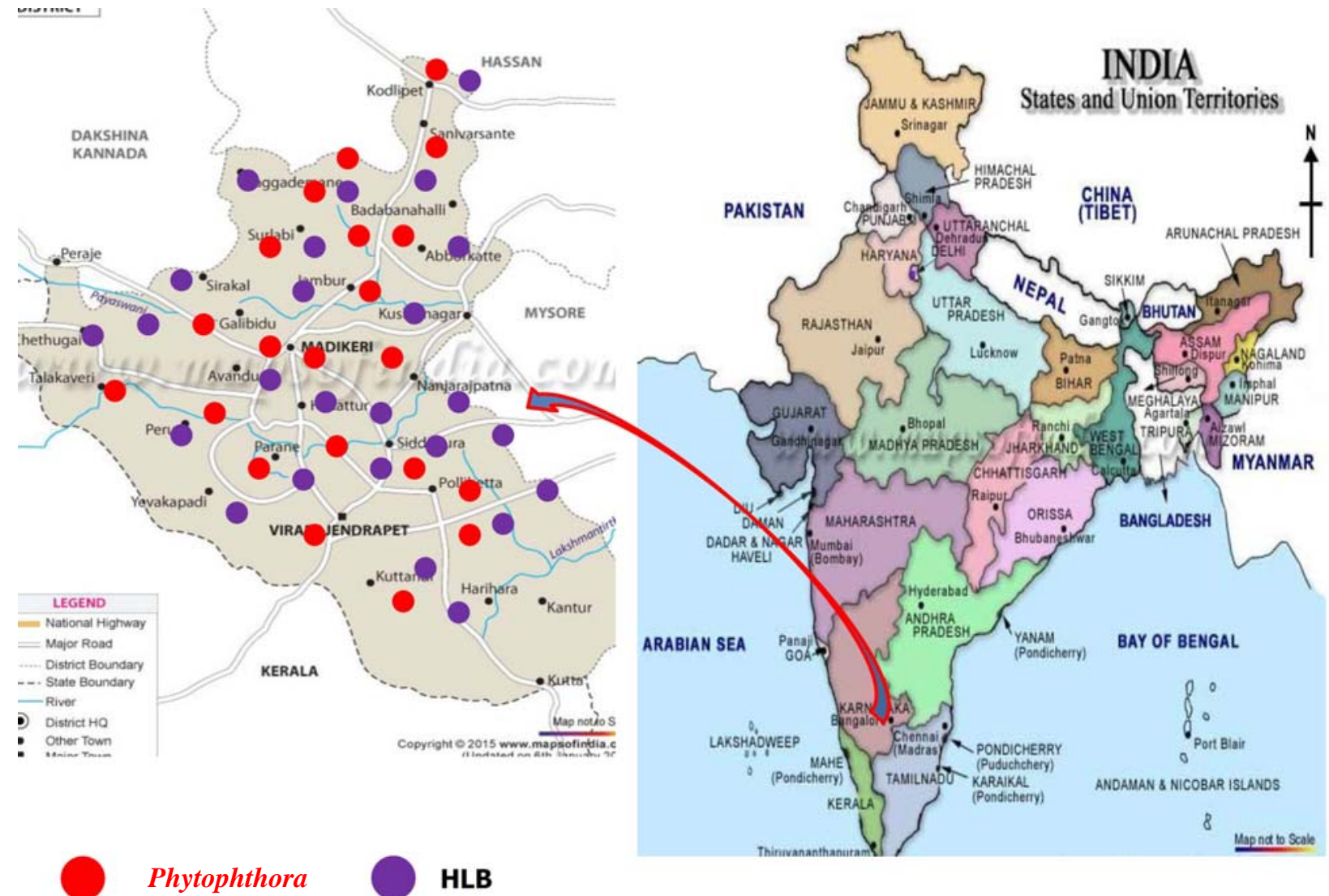

Fig. 1 Survey conducted in different places of Coorg region in Karnataka. 
incidence of Phytophthora and HLB disease on Coorg mandarin (plants raised from true seeds and grafted on Rangpur lime rootstock).

The disease incidence was estimated in each field by visual examination of plants by making two diagonal transects across the field in the form of an "X" (50 plants along each diagonal). In each orchard, 100 trees per orchard were rated for foot rot and gummosis lesion severity on a scale of 0 (no lesion) to 5 (entire trunk covered with lesion and gummosis). The incidence was determined as the proportion of plants showing symptoms of Phytophthora (root rot, discoloration of the bark surface, discoloration of the underlying tissues, dieback, dried the whole part of the plant and exudation of gum from infected tissues) and HLB (mottling and yellowing) expressed as a percentage of the total number of plants assessed. The rhizosphere soil and infected root and plant parts were also collected from surveyed areas to study occurrence and distribution of Phytophthora species. Similarly, HLB disease incidence was rated by using three-point scale with 0 representing no symptom observed on plant canopy, $1=$ mild (from $1 \%$ to $30 \%$ of the canopy), $2=$ moderate (from $31 \%$ to $50 \%$ of the canopy), $3=$ severe (more than $50 \%$ of the canopy). Percentage of disease incidence of Phytophthora and HLB was calculated based on the number of observed symptomatic plants over the total number of the assessed plants. The percentage of disease incidence (PDI) was estimated using Eq. (1).

$$
\begin{aligned}
& \text { PDI } \\
& (\%)
\end{aligned}=\frac{\text { Total infected citrus trees }}{\text { Total number of trees observed }} \times 100
$$

Disease severity index (DSI) was then calculated as Eq. (2):

$$
\begin{aligned}
& \mathrm{DSI} \\
& (\%)
\end{aligned}=\frac{X_{1}+X_{2}+\ldots+X_{n}}{Y \times \text { maximum rating scale }} \times 100
$$

where, $X=$ sum score of disease severity of each citrus plant; $Y=$ total number of plant assessed.

\subsection{Collection of Disease Samples of Phytophthora and $H L B$}

A total of 177 Phytophthora infected plant roots
(59), soil (118) and 576 greening or HLB infected Coorg mandarin samples were collected from 184 Coorg mandarin orchards, showing typical symptoms of Phytophthora infection, greening, yellowing and mottling in different places of Coorg and Hassan, and one sample from each location without any symptoms on Coorg mandarin plants was also collected.

\subsection{Isolation of Phytophthora Species from Plant Parts and Soil}

\subsubsection{Coorg Mandarin Infected Plant Parts}

Phytophthora infected Coorg mandarin plant parts (twigs, trunk and gummosis part) were collected from different orchards in Coorg region. The collected samples which may have been contaminated with different pathogens were removed by surface sterilization using standard protocol [11]. Then samples were aseptically placed on specific media amended with pimaricin, ampicillin, rifamficin, pentachloronitrobenzene (PCNB) and hymexazol (RPARH) medium.

2.3.2 Soil from the Rhizosphere of Infected and Healthy Plants

The soil sample collected from rhizosphere (1 gm) of infected and healthy plants were thoroughly mixed in $10 \mathrm{~mL}$ of sterile distilled water, and $1 \mathrm{~mL}$ each of soil suspensions was transferred into specific containing Petri plates and incubated at $25 \pm 2{ }^{\circ} \mathrm{C}$ for $48 \mathrm{~h}$. Later, the media on Petri plate was washed with sterile distilled water to observe growth of Phytophthora culture. The colony resembling Phytophthora were picked and transferred to specific media. Stock cultures were maintained on V8 agar. The pure culture of isolated Phytophthora isolates was used for morphological studies (colony growth, sporangia, oogonia, antheridia, chlamydospores, hyphal swellings and aggregations with species descriptions in the literature) using light microscope (Nikon Eclipse 50i) at $400 \times$ magnification. The images were imported to ImageJ (National Institutes 
of Health, USA) and analyzed by setting the scale at 3.6 pixels/ $\mu \mathrm{m}$. Radial growth was calculated by placing a mycelial plug on fresh (RPARH) media, and incubating for three days at room temperature under continuous fluorescent light. Three plates per isolate were prepared. Colony pattern was characterized according to morphology for 7-day-old colonies [12]. Mycelial growth was classified as reduced or appressed, scanty-fluffy or densely-fluffy following the descriptions described by Erwin and Ribeiro [12].

Pathogenicity test: To confirm pathogenicity of casual agent, the un-infected healthy three-month-old seedlings of Coorg mandarins were inoculated with sporangial suspension at a concentration of $1 \times 10^{6}$ conidia/mL by making the wounded (pin prick method) at crown region of the plant. The inoculated Coorg mandarin plants were kept under controlled condition to monitor the expression of wilting or rotting, gummosis and lesions symptoms. Subsequently, pathogen was re-isolated from the diseased plant showing gummosis and lesions development symptoms on crown region of the plant, confirming Koch's postulates. The test was repeated twice.

\subsection{Molecular Characterization}

\subsubsection{Fungal Genomic DNA Isolation}

The pure culture of the fungus was grown on potato dextrose broth at $25 \pm 2{ }^{\circ} \mathrm{C}$ for $7 \mathrm{~d}$. The fungal mycelium was harvested by filtration through Whatman No.1 filter paper and washed with sterile distilled water and dried. Two grams of dried mycelium were used for total genomic DNA isolation by following modified protocol of CTAB method [13]. The quality of the genomic DNA was checked on $0.8 \%$ agarose gel and stored at $-20{ }^{\circ} \mathrm{C}$ till further use. To confirm identity of the pathogen, Total genomic DNA was amplified by PCR using universal internal transcriber spacer region primers ITS1 (5'-TCCGTAGGTGAACCTGCGG-3') and ITS4 (5'-TCCTCCGCTTATTGATATGC-3') [14]. The DNA amplification was performed with 35 cycles of denaturation for $1 \mathrm{~min}$ at $94{ }^{\circ} \mathrm{C}$, primer annealing for $45 \mathrm{~s}$ at $55{ }^{\circ} \mathrm{C}$ for Phytophthora and primer extension for $90 \mathrm{~s}$ at $72{ }^{\circ} \mathrm{C}$, with an initial denaturation at $94{ }^{\circ} \mathrm{C}$ for $3 \mathrm{~min}$ and a final extension for $10 \mathrm{~min}$ at $72^{\circ} \mathrm{C}$. The PCR reactions were carried out in a GeneAmp PCR system 9700 (PE Applied Biosystems, Foster City, CA) thermocycler. All amplifications were performed in volumes of $25 \mu \mathrm{L}$ PCR mix containing 2 $\mu \mathrm{L}$ DNA templates, $1.5 \mathrm{U}$ Taq DNA polymerase, 25 $\mathrm{mM} \mathrm{MgCl} 2,2 \mathrm{mM}$ dNTPs and 20 pmol of each primer. PCR products were electrophoresed $(1 \mathrm{~h}$ at $80 \mathrm{~V})$ in $0.8 \%$ agarose gels in Tris-Borate-EDTA buffer, $\mathrm{pH} 8$. Gels were stained with ethidium bromide $(10 \mathrm{mg} / \mathrm{mL})$ and viewed in a gel documentation system (Alpha Innotech, USA).

\subsubsection{HLB Disease}

The total nucleic acid was isolated from Coorg mandarin samples (576 HLB infected) by using CTAB method [13]. Finally, DNA pellets were dried under vacuum, resuspended in $50 \mu \mathrm{L}$ of TE buffer (10 $\mathrm{mM}$ Tris-HCl, $\mathrm{pH}$ 7.5; $1 \mathrm{mM}$ EDTA, $\mathrm{pH}$ 8.0) and maintained at $-20{ }^{\circ} \mathrm{C}$ until being used. The presence of HLB in 540 samples was confirmed by PCR using 16S rRNA gene specific universal primers [15]. The DNA amplification was performed with 35 cycles as described above, except primer annealing for $45 \mathrm{~s}$ at $56{ }^{\circ} \mathrm{C}$ for HLB. The amplified nested PCR products HLB (1.2 kbp) and Phytophthora (550 bp) was purified from agarose gels using the QIAquick gel extraction kit (Qiagen, Hilder, Germany) and cloned into $\mathrm{pTZ57R/T}$ cloning vector according to manufacturer's directions (MBI Fermentas, Germany). The transformation was performed using Escherichia coli (DH5 $\alpha$ ) cells. The transformed plasmid was isolated using Qiagen plasmid miniprep kit (Qiagen, Hilder, Germany) and sequenced using automated DNA sequencing facility at Eurofins Genomics India Pvt. Ltd., Bangalore, India.

\subsubsection{Sequence Analysis}

The sequences of HLB (1.2 kbp) and Phytophthora (550 bp) were subjected to NCBI BLAST to search 
for similar sequences in the database. The related sequences obtained from the database belonging to different Liberobacter and Phytophthora species infecting diverse hosts were used for analysis (Tables 1 and 2). Sequences were aligned using MUCSLE method implemented in SEAVIEW program [16]. The nucleotide (nt) sequence identity matrixes for the HLB and Phytophthora were generated using BioEdit sequence alignment editor (version 5.0.9) [17]. The phylogenetic tree was constructed by neighbor joining method using MEGA 6.0.1 version software [18] with 1,000 bootstrapped replications to estimate evolutionary distances between all pairs of sequences simultaneously.

Table 1 Phytophthora species sequence accession numbers obtained from GenBank database used in the analyses.

\begin{tabular}{|c|c|c|c|}
\hline Phytophthora species & Accession number & Phytophthora species & Accession number \\
\hline P. nicotianae-NRCPh-1 & HM807369 & P. palmivora-NRCPh-15 & JF792543 \\
\hline P. nicotianae-NRCPh-2 & JF792525 & P. palmivora-NRCPh-20 & JF792544 \\
\hline P. nicotianae-NRCPh-3 & JF792526 & P. palmivora-NRCPh-21 & JF792545 \\
\hline P. nicotianae-NRCPh-4 & HM807370 & P. palmivora-NRCPh-23 & JF792546 \\
\hline P. nicotianae-NRCPh-5 & JF792527 & P. palmivora-NRCPh-27 & JF792547 \\
\hline P. nicotianae-NRCPh-6 & HM807371 & P. palmivora-NRCPh-29 & JF792548 \\
\hline P. nicotianae-NRCPh-7 & HM807372 & P. palmivora-NRCPh-79 & JX965378 \\
\hline P. nicotianae-NRCPh-9 & JF792528 & P. palmivora-NRCPh-94 & JN559844 \\
\hline P. nicotianae-NRCPh-1 & JF792529 & P. palmivora-NRCPh-100 & KF010299 \\
\hline P. nicotianae-NRCPh-17 & JF792530 & P. palmivora & KR920754 \\
\hline P. nicotianae-NRCPh-18b & JF792531 & P. palmivora & JX198553 \\
\hline P. nicotianae-NRCPh-19 & JF792532 & P. palmivora & KR920757 \\
\hline P. nicotianae-NRCPh-22 & JF792533 & P. palmivora & JX198562 \\
\hline P. nicotianae-NRCPh-24 & JF792534 & P. palmivora & KP183963 \\
\hline P. nicotianae-NRCPh-31 & JF792535 & P. palmivora & KR920758 \\
\hline P. nicotianae-NRCPh-37 & JF792536 & P. palmivora & HQ237479 \\
\hline P. nicotianae-NRCPh-41 & JF792537 & P. palmivora & KR920760 \\
\hline P. nicotianae-NRCPh-52 & JF792538 & P. palmivora & JF792548 \\
\hline P. nicotianae-NRCPh-56 & JF792539 & P. palmivora & KU877819 \\
\hline P. nicotianae-NRCPh-58 & JX965375 & P. palmivora & AB669142 \\
\hline P. nicotianae-NRCPh-61 & JX965375 & P. palmivora & AF266780 \\
\hline P.nicotianae-NRCPh-66 & JX965375 & P. citrophthora-NRCPh-97 & JN559845 \\
\hline P. nicotianae-NRCPh-70 & JX965375 & P. citrophthora & GU993889 \\
\hline P.nicotianae-NRCPh-71 & JX965376 & P. citrophthora & HQ643208 \\
\hline P. nicotianae-NRCPh-76 & JX965377 & P.citrophthora & HQ643207 \\
\hline P. nicotianae-NRCPh-81 & JX965379 & P. citrophthora & HQ261535 \\
\hline P. nicotianae-NRCPh-89 & JN559843 & P. citrophthora & AF266785 \\
\hline P. nicotianae-NRCPh-98 & JN559846 & P. capsici & AF266787 \\
\hline P. nicotianae-NRCPh-99 & JX965380 & P. cinnamomi & AF266764 \\
\hline P. nicotianae & KJ549640 & P. insolita & AF271222 \\
\hline P. nicotianae & GU111669 & P. inundata & AY659717 \\
\hline P. nicotianae & KJ549641 & P. iротоеае & AY770742 \\
\hline P. nicotianae & KJ494919 & & \\
\hline P. nicotianae & KJ494917 & & \\
\hline P. nicotianae & KJ494914 & & \\
\hline P. nicotianae & KJ494913 & & \\
\hline P. nicotianae & KJ494911 & & \\
\hline P. nicotianae & AF266776 & & \\
\hline
\end{tabular}



Decline in Coorg Mandarin in India

Table 2 Ca. Liberibacter species sequence accession numbers obtained from GenBank database used in the analyses.

\begin{tabular}{|c|c|c|c|}
\hline Ca. Liberibacter species & Accession number & Ca. Liberibacter species & Accession number \\
\hline Ca. L. asiaticus-MPW1456 & AB558580 & Ca. L. asiaticus-H22Y & JQ867419 \\
\hline Ca. L. asiaticus & AB480072 & Ca. L. asiaticus & EU224393 \\
\hline Ca. L. asiaticus & DQ471900 & Ca. L. asiaticus-F11Dade & EU130552 \\
\hline Ca. L. asiaticus-GFB-Selangor & EU224393 & Ca. L. asiaticus-H36YPENINSULAR & JQ867409 \\
\hline Ca. L. asiaticus & EU130552 & Ca. L. asiaticus strain ACL & KC551941 \\
\hline Ca. L. asiaticus & DQ157275 & Ca. L. asiaticus clone $33 \mathrm{a}$ & JX430434 \\
\hline Ca. L. asiaticus & DQ157273 & Ca. L. asiaticus & AB555706 \\
\hline Ca. L. asiaticus & DQ303210 & Ca. L. asiaticus & DQ157274 \\
\hline Ca. L. asiaticus & AY919311 & Ca. L. asiaticus & JQ867445 \\
\hline L. Phytophthora Okinawa-7 & AB008366 & Ca. L. asiaticus & KU761591 \\
\hline Ca. L. asiaticus & KJ944269 & Ca. L. asiaticus- $\mathrm{H} 23 \mathrm{Y}$ & JQ867420 \\
\hline Ca. L. asiaticus & KJ944267 & Ca. L. asiaticus-H16Y & JQ867418 \\
\hline Ca. L. asiaticus & KJ944262 & Ca. L. asiaticus-H15QR 1 & JQ867447 \\
\hline Ca. L. asiaticus-TNal-9 & KC800958 & Ca. L. asiaticus- $\mathrm{H} 31 \mathrm{Y}$ & JQ867425 \\
\hline Ca. L. asiaticus-TNal-8 & KC800957 & Ca. L. asiaticus-Polibetta & FJ827779 \\
\hline Ca. L. asiaticus-TNmal-2 & KC800951 & Ca. L. asiaticus-Guangxi-GL-19-CHN & EU921616 \\
\hline Ca. L. asiaticus isolate Naga-1 & KC119094 & Ca. L. asiaticus-Guangxi-GL-7-CHN & EU921614 \\
\hline Ca. L. asiaticus-H13QR & JQ867445 & $\begin{array}{l}\text { Ca. L. asiaticus-Guangxi-STY partial } \\
\text { sequence }\end{array}$ & DQ432000 \\
\hline Ca. L. asiaticus-CAHLB2012 & JX455745 & Ca. L. asiaticus-Guangdong-PG & DQ431999 \\
\hline Ca. L. asiaticus-Umiam-2 & JX284240 & Ca. L. americanus & AY742824 \\
\hline Ca. L. asiaticus-gj1 & JN049632 & Ca. L. americanus-SaoPaulo-40 & EU921625 \\
\hline Ca. L. asiaticus & EU265646 & Ca. L. americanus-SaoPaulo-275 & EU921623 \\
\hline Ca. L. asiaticus-MH & JQ900232 & Ca. L. americanus-SaoPaulo-PW-49 & EU921624 \\
\hline Ca. L. asiaticus-MDL1394-DR002-8 & FJ821713 & Ca. L. americanus-LJZ-5110 & FJ263689 \\
\hline $\begin{array}{l}\text { Ca. L. asiaticus isolate } \\
\text { MDL1391-DR002-5 }\end{array}$ & FJ821710 & Ca. L. americanus-LJZ-5025 & FJ263695 \\
\hline Ca. L. asiaticus & EU224394 & Ca. L. americanus-LJZ-5020 & FJ263694 \\
\hline Ca. L. asiaticus-F17PalmBeach & EU130554 & Ca. L. americanus-LJZ-5130 & FJ263692 \\
\hline Ca. L.-Kinnow & LN835770 & Ca. L. americanus-LJZ-5128 & FJ263691 \\
\hline Ca. L. asiaticus & KM224448 & Ca. L. americanus-LJZ-5135 & FJ263693 \\
\hline Ca. L. asiaticus clone $\mathrm{C} 2$ & KM224447 & Ca. L. americanus-LJZ-5115 1 & FJ263690 \\
\hline Ca. L. asiaticus-Mmnd-1 & KC800962 & Ca. L. europaeus & JX244259 \\
\hline Ca. L. asiaticus-TNal-7 & KC800956 & Ca. L. europaeus-Psy6 & JX244258 \\
\hline Ca. L. asiaticus-TNal-5 & KC800954 & Ca. L. africanus & KX990287 \\
\hline Ca. L. asiaticus-ACL-Katol-3 & KC551941 & Ca. L. africanus & KX990288 \\
\hline Ca. L. asiaticus-H20QR & JQ867450 & Ca. L. africanus & KY000562 \\
\hline Ca. L. asiaticus-H18QR & JQ867448 & Ca. L. africanus & KY000560 \\
\hline Ca. L. asiaticus-H12QR & JQ867444 & Ca. L. psyllaurous-Tx15 & EU812556 \\
\hline Ca. L. asiaticus-11QR & JQ867443 & Ca. L. psyllaurous-Tom 100 & EU812558 \\
\hline Ca. L. asiaticus-Florida-8 & EU921617 & Ca. L. psyllaurous-PRR1 & EU812559 \\
\hline Ca. L. asiaticus-Jiangxi-GC & DQ432003 & Ca. L. solanacearum & EU935004 \\
\hline Ca. L. asiaticus-Chongqing-ZG & DQ432004 & & \\
\hline Ca. L. asiaticus-H34Y & JQ867427 & & \\
\hline Ca. L. asiaticus & KJ944265 & & \\
\hline Ca. L. asiaticus-H2C & JQ867437 & & \\
\hline Ca. L. asiaticus-H35C & JQ867434 & & \\
\hline Ca. L. asiaticus-AL-Kahikuchi & KC551939 & & \\
\hline
\end{tabular}




\section{Results}

\subsection{Survey}

\subsubsection{HLB Disease}

A systematic random survey, conducted in 184 orchards of Kodagu district, revealed that the disease incidence of HLB was more severe in most of the Coorg mandarin plants. The disease incidence ranged from $35 \%$ to $64.2 \%$ on Coorg mandarin plants raised from true seeds and grafted plants growing in commercial groves in the Kodagu district, Karnataka state of India (Table 3). No Coorg mandarin plants (raised from true seeds or budded) displayed a consistently low or high level HLB incidence in every grove sampled. Further, it was also observed that the occurrence of yellowing was more in budded plants after six years of planting than the plant raise from true seeds. The infected plants showed symptoms of sparse foliage, very short twigs with narrow leaves characterized with yellowish colored leaves similar to zinc deficiency. Mottled leaves and fruits with aborted seeds provided good indication of greening disease in all surveyed areas, both in true seeds and grafted Coorg mandarin plants (Fig. 2). The severity of disease increased proportionally in orchards wherein true seed plants were more than 10 years old and budded plants were more than six years in age (Table 3).

\subsubsection{Phytophthora Disease}

A systematic random survey for incidence and severity of Phytophthora on Coorg mandarin plants revealed the occurrence of disease in all surveyed orchards with different magnitude of infection (Table 4). Incidence of the disease was not found to be

Table 3 Citrus greening bacteria infecting Coorg mandarin samples was collected from Kodagu and Hassan.

\begin{tabular}{|c|c|c|c|c|c|c|c|}
\hline Place & $\begin{array}{l}\text { No. of } \\
\text { orchards }\end{array}$ & $\begin{array}{l}\text { Age group } \\
\text { (years) }\end{array}$ & $\begin{array}{l}\text { No. of samples } \\
\text { collected }\end{array}$ & $\begin{array}{l}\text { Type of } \\
\text { symptoms }\end{array}$ & $\begin{array}{l}\text { Greening } \\
\text { (HLB) }\end{array}$ & & $\begin{array}{l}\text { No. of samples PCR } \\
\text { positive for greening }\end{array}$ \\
\hline & & & & & Av. PDI (\%) & DSI (\%) & \\
\hline Madikeri & 48 & $8-15$ & 165 & $\begin{array}{l}\text { Only yellowing, } \\
\text { yellowing and } \\
\text { mottling }\end{array}$ & 61.56 & 4.1 & 152 \\
\hline Somawarpet & 70 & $5-15$ & 230 & $\begin{array}{l}\text { Only yellowing, } \\
\text { yellowing and } \\
\text { mottling }\end{array}$ & 64.20 & 3.1 & 206 \\
\hline Virajpet & 58 & $8-15$ & 161 & $\begin{array}{l}\text { Only yellowing, } \\
\text { yellowing and } \\
\text { mottling }\end{array}$ & 57.96 & 3.6 & 146 \\
\hline Belur (Hassan) & 8 & $4-5$ & 20 & $\begin{array}{l}\text { Yellowing and } \\
\text { mottling }\end{array}$ & 35.00 & 2.0 & 19 \\
\hline Total & 184 & & 576 & & & & 523 \\
\hline
\end{tabular}

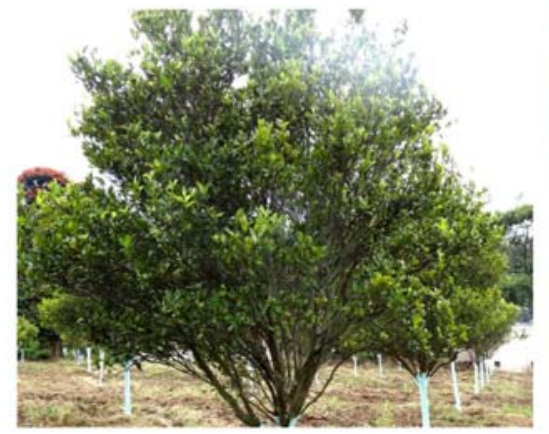

Over view of the field

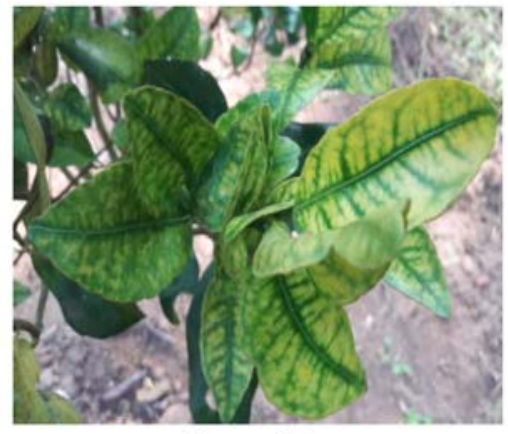

Mottling of leaves

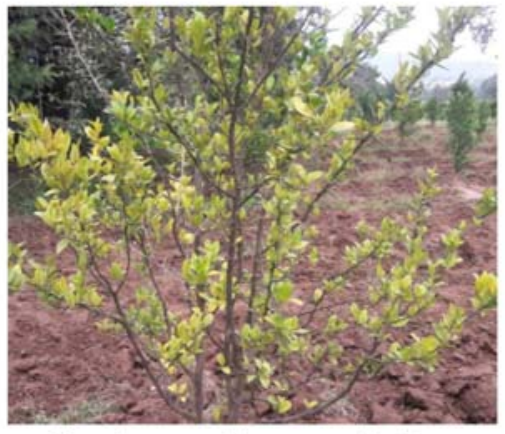

Yellowing and sparse foliage

Fig. 2 Coorg mandarin plant showing yellowing, mottling and complete yellowing and greening symptoms under natural conditions. 
Decline in Coorg Mandarin in India

Table 4 Phytophthora infecting Coorg mandarin samples was collected from Kodagu and Hassan.

\begin{tabular}{|c|c|c|c|c|c|c|c|c|}
\hline Place & $\begin{array}{l}\text { No. of } \\
\text { orchards }\end{array}$ & $\begin{array}{l}\text { Age group } \\
\text { (years) }\end{array}$ & $\begin{array}{l}\text { No. of } \\
\text { samples } \\
\text { collected }\end{array}$ & $\begin{array}{l}\text { No. of } \\
\text { samples } \\
\text { collected }\end{array}$ & $\begin{array}{l}\text { Type of } \\
\text { symptoms }\end{array}$ & Phytophthorc & & $\begin{array}{l}\text { No. of samples } \\
\text { positive to } \\
\text { Phytophthora }\end{array}$ \\
\hline & & & Root & Soil & & Av. PDI (\%) & DSI $(\%)$ & \\
\hline Madikeri & 48 & $8-15$ & 10 & 23 & $\begin{array}{l}\text { Foot rot, wilting, } \\
\text { gummosis }\end{array}$ & 35.62 & 2.5 & 23 \\
\hline Somwarapet & 70 & $5-15$ & 20 & 52 & $\begin{array}{l}\text { Lesions in collar } \\
\text { region, foot rot, } \\
\text { wilting, gummosis }\end{array}$ & 35.34 & 3.2 & 45 \\
\hline Virajpet & 58 & $8-15$ & 25 & 33 & $\begin{array}{l}\text { Lesions in collar } \\
\text { region, foot rot, } \\
\text { wilting, gummosis }\end{array}$ & 31.42 & 2.57 & 34 \\
\hline Belur (Hassan) & 8 & $4-5$ & 4 & 10 & Foot rot, wilting & 22.50 & 1.0 & 9 \\
\hline Total & 184 & & 59 & 118 & & & & 111 \\
\hline
\end{tabular}

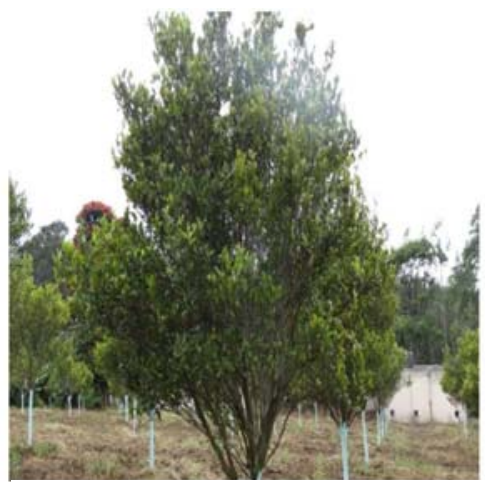

Normal

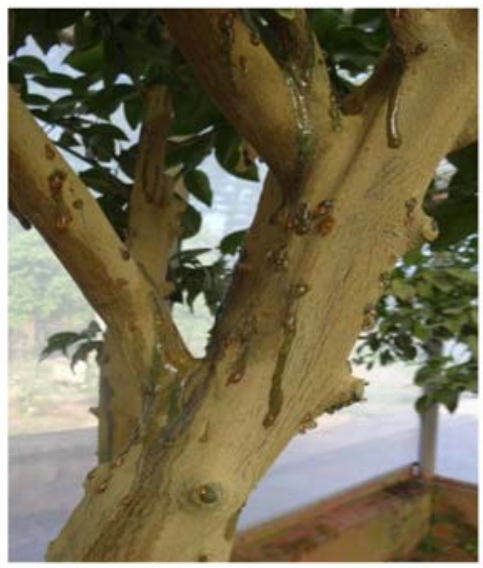

Gummosis

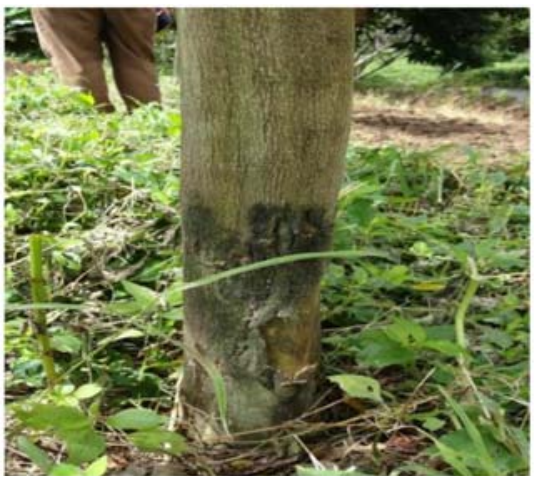

Foot rot

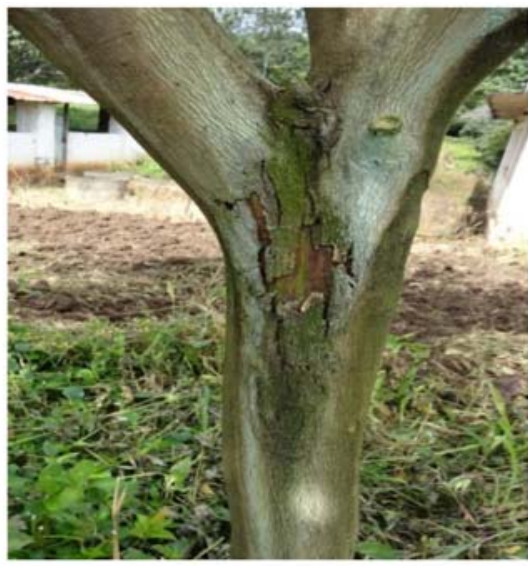

Trunk slitting

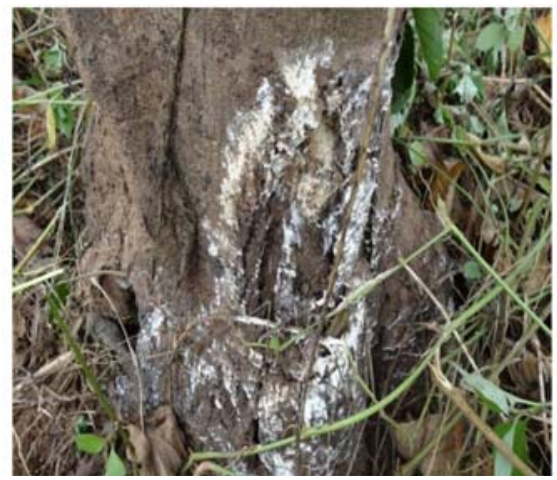

Mycelia growth on collar region

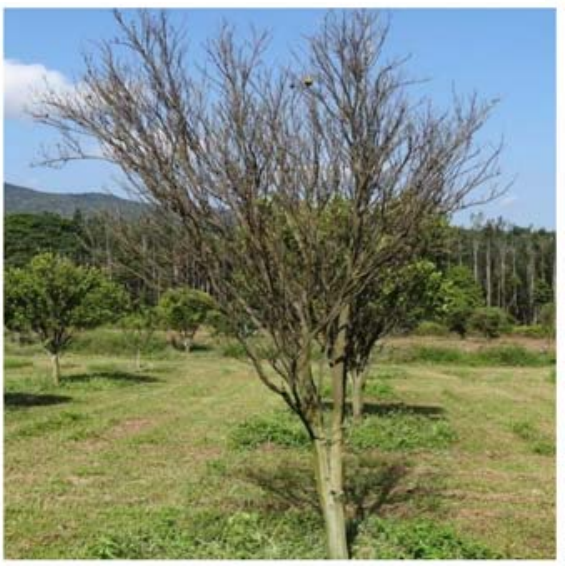

Complete dead

Fig. 3 Coorg mandarin plant showing foot rot, gummosis, collar rot and trunk slit.

affected by seasons, which could be attributed to the limited fluctuations in ambient temperatures and relative humidity. In post-monsoon season, the main symptoms observed in different orchards were rotting of the rootlets, leading to disintegration of the cortical tissue on feeder roots, with a white thread-like stele (inner tissue of the fibrous root). During summer season, heavy gum oozing was observed on higher branches irrespective of age of the plants. As the disease progressed, bark shrinks, cracks, shredding in lengthwise direction exposing black discolored internal tissue. Under severe infection, plants blossom heavily and die after fruit mature (Fig. 3).

Foot rot infection was recorded especially in 
budded plants wherein the bud union lied close to the soil line promoting buildup of Phytophthora populations and increased risk of foot rot infection. Crown rot resulted from bark infection below the soil line when susceptible rootstocks were used. Incidence of Phytophthora disease was similar in true seeds and grafted Coorg mandarin plants in all surveyed areas. The highest incidence of Phytophthora disease was recorded in Madikeri (35.62\%) with DSI rating 2.5, followed by Somwarapet and the lowest in Hassan (22.5\%) with DSI rating 1 (Table 4).

\subsection{Morphological Characterization}

Out of 177 Phytophthora isolates, 111 were characterized from 33 places of Kodagu district. Among these, 21 isolates were isolated from symptomatic plant roots ( $P$. palmivora and $P$. nicotianae). Fifty-eight (58) isolates belonging to $P$. palmivora, were isolated from $60.89 \%$ of soil samples and 32 isolates belonging to $P$. nicotianae, were isolated from $41.55 \%$ of soil samples, respectively (Fig. 4).

The pathogen was isolated on specific media, the pure cultures of Phytophthora isolates were maintained in V8 slants for further use. On the basis of colony growth patterns coupled with microscopic features of morphological structures, the isolates were first grouped in five distinct morphotypes as described by Erwin and Ribeiro [12] (data not shown). Later, the isolates were finalized to two morphotypes, which were identified as $P$. palmivora and P. nicotianae.

\subsubsection{Phytophthora palmivora}

P. palmivora produced papillate sporangia with large chlamydospores, which could be easily distinguished from P. citrophthora and P. nicotianae by the caducous sporangia. Abundant sporangia were observed $5 \mathrm{~d}$ after inoculation and were typically papillate with short pedicels. Chlamydospores were spherical to ellipsoid structures with a thick cell wall $(4 \mu \mathrm{m})$ and a diameter of $35-45 \mu \mathrm{m}$. Most of the isolates were heterothallic. Antheridia were amphigynous and spherical or oval and oogonia were smooth, spherical and 15-64 $\mu \mathrm{m}$ in diameter.

\subsubsection{Phytophthora nicotianae}

Tufted colony morphology and an arachnoid branching mycelium were the typical characteristic of $P$. nicotianae. Sporangia were more regular, symmetrical with a single apex, non-caducous, ellipsoid or ovoid or pyriform to spherical sporangia with usually a single papillum [12] and it could be easily

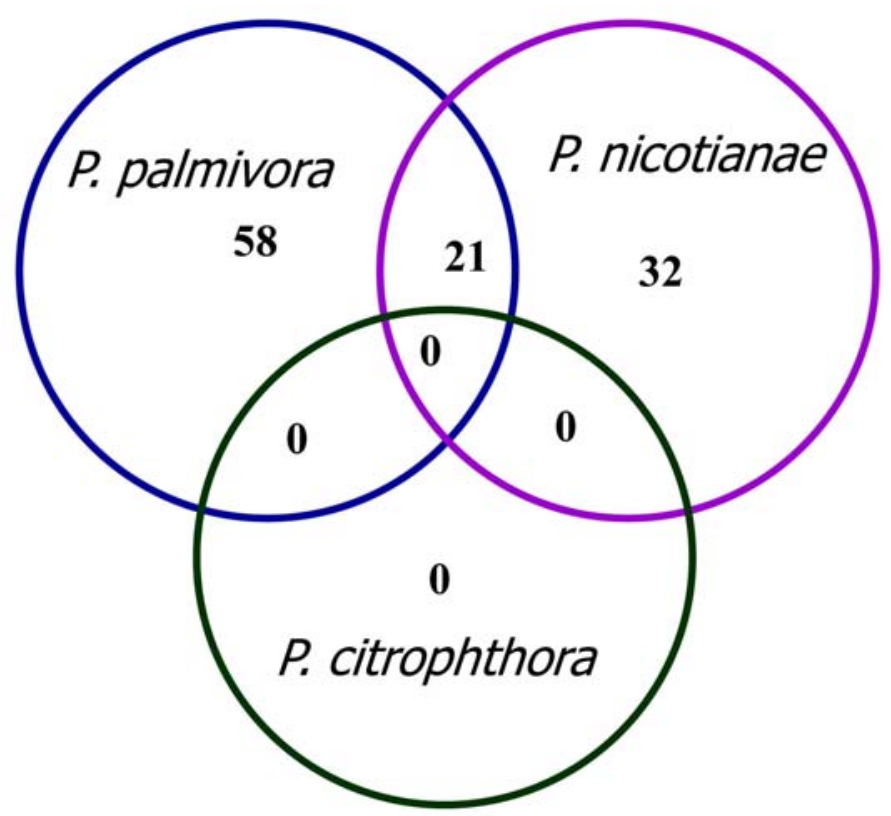

Fig. 4 Percentage of samples showing infection of different Phytophthora species. 
differentiated from the caducous sporangia of $P$. palmivora.

\subsection{Molecular Characterization}

\subsubsection{Phytophthora}

Out of 111 Phytophthora isolates, 52 representative isolates were chosen from the morphological groups for molecular analysis. The total genomic DNA of 52 Phytophthora isolates was amplified by PCR using universal ITS region specific primers. The expected PCR amplicon size of $550 \mathrm{bp}$ was amplified (data not shown), which corresponds to ITS region of the fungi. The amplified PCR product was cloned and sequenced. The sequence analysis showed that 30 of 52 Phytophthora isolates belonging to $P$. palmivora with nt identity more than $98 \%$ (data not shown) remaining 10 isolates (FG7, FG8, FG9, FG10, FG11, FG12, FG30, FG34, FG38, FG41) with varied nt identity (70.6\%-97.4\%) were selected for further analysis. Similarly, eight isolates belong to $P$. nicotianae, showed nt identity more than $97 \%$ among themselves (data not shown) of these four isolates (FG16, FG17, FG18, FG20) were selected with varied nt identity (91.6\%-100\%) for further analysis. The sequences of 10 representative $P$. palmivora and four $P$. nicotianae isolates were available in database under following accession number (Table 5).

The comparative ITS region analysis between 10 representative $P$. palmivora and four $P$. nicotianae isolates infecting Coorg mandarin showed less than $80 \%$ nt identity. Further, the ITS sequences of $P$. palmivora and $P$. nicotianae isolates infecting Coorg mandarin were compared with other citrus infecting species, viz., P. palmivora (21 isolates), P. nicotianae (37 isolates), $P$. citrophthora (six isolates) and Phytophthora spp. (five isolates) infecting different crops available in the database (Table 1). The analysis showed that all 10 representative $P$. palmivora isolates (FG7, FG8, FG9, FG10, FG11, FG12, FG30, FG34, FG38 and FG41) isolated from Coorg mandarin shared nt identity range from $87.6 \%$ to $100 \%$ with $P$. palmivora isolates, $<75 \%$ nt identity with $P$. nicotianae isolates and $<70 \%$ nt identity with $P$. citrophthora isolates available in the NCBI database infecting citrus in India. Similarly, $P$. nicotianae isolates (FG16, FG17, FG18 and FG20) were analyzed. The analysis showed that $P$. nicotianae isolates shared the nt identity ranging from $92.4 \%$ to $100 \%$ with $P$. nicotianae isolates infecting citrus, less than $75 \% \mathrm{nt}$ identity with $P$. palmivora and $P$. citrophthora isolates infecting citrus, respectively. The phylogenetic tree based on comparison of ITS analysis of 10 representative $P$. palmivora and four $P$. nicotianae isolates infecting Coorg mandarin with other citrus infecting $P$. palmivora (21), $P$. nicotianae (37), $P$. citrophthora (6) and other Phytophthora spp. (5). The analysis showed that all $P$. palmivora, $P$. nicotianae isolates infecting Coorg mandarin clustered with respective isolates of $P$. palmivora and $P$. nicotianae infecting citrus in India (Fig. 5).

\subsubsection{HLB Disease}

Total 576 infected leaf samples of Coorg mandarin were collected from 184 Coorg mandarin orchards, showing typical symptoms of greening, yellowing and mottling. The total DNA isolated from 576 samples of Coorg mandarin was amplified by PCR using $16 \mathrm{~S}$ rRNA gene specific primers. And 523 of 576 samples were confirmed the presence of HLB infection, with expected PCR amplicon size of 1.2 bp being amplified (data not shown) from all samples. The sequencing of representative samples showed a match with previously identified citrus greening bacteria. Therefore, only six (CGB1, CGB1a, CGB2 CGB3 CGB4 and CGB5) out of 523 samples were selected for cloning and sequencing, based on symptom severity and location. The 16S rRNA gene sequence analysis showed that six HLB Coorg mandarin isolates shared nt identity ranging from $94.3 \%$ to $98.9 \%$ among themselves. Further, HLB Coorg mandarin isolates $16 \mathrm{~S}$ rRNA gene was compared with other Ca. L. asiaticus (65 isolates), Ca. L. americanus (11 isolates), Ca. L. africanus (four isolates), Ca. L. psyllaurous (three 


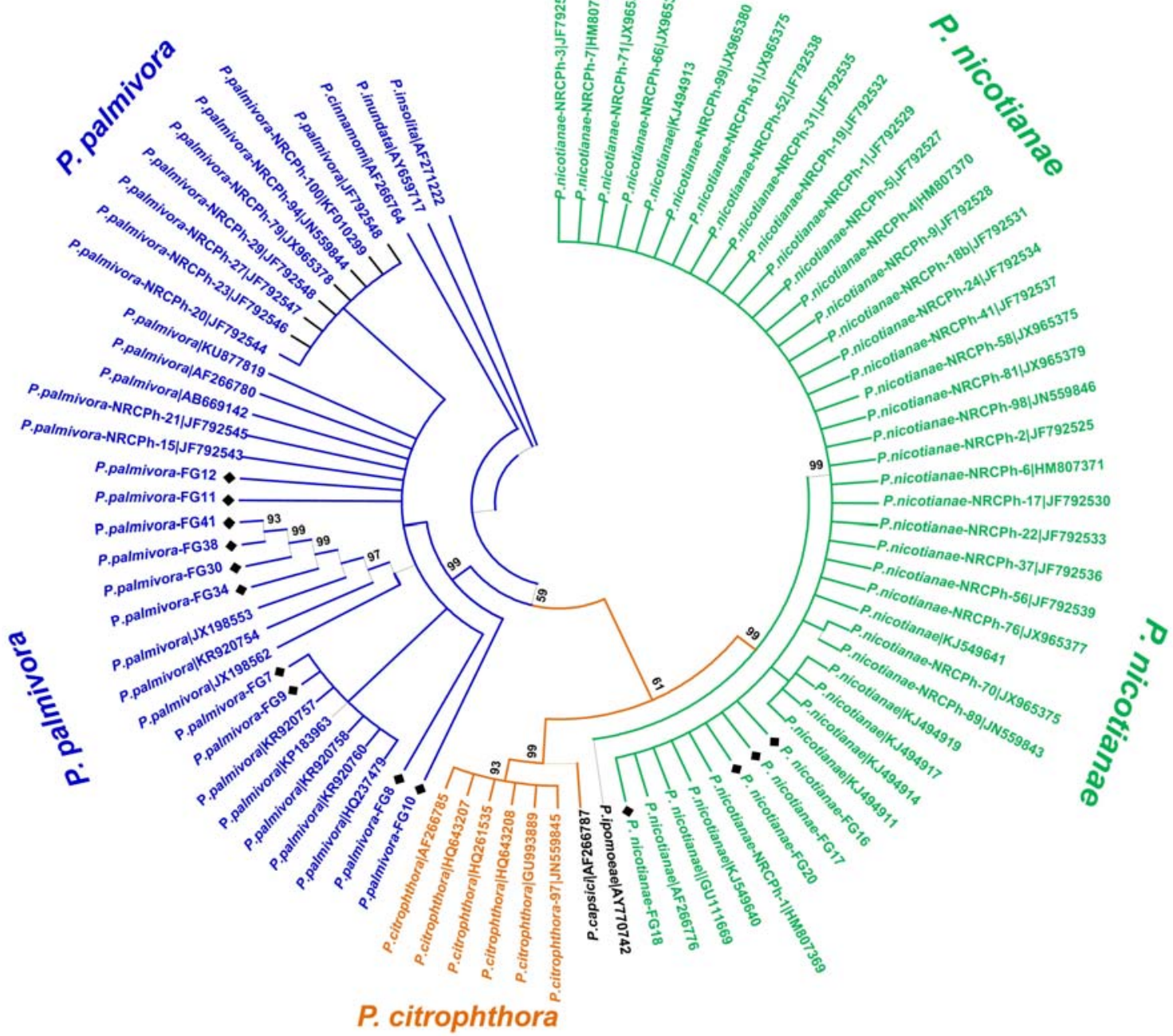

Fig. 5 Phylogenetic analysis of Phytophthora species infecting Coorg mandarin using rDNA ITS using neighbor-joining algorithm.

Horizontal distances are proportional to sequence distances; vertical distances are arbitrary. The trees are unrooted. A bootstrap analysis with 1,000 replicates was performed and the bootstrap percent values more than 50 are numbered along branches.

isolates), Ca. L. solanacearum (one isolate) retrieved from GenBank database. The analysis showed that three HLB Coorg mandarin isolates (CGB1, CGB1a, CGB3) shared nt identity ranging from $94.4 \%$ to 95.3\% with Ca. L. asiaticus (AB008366) isolate infecting citrus in Asia, one HLB isolate (CGB2) shared nt identity of $96.9 \%$ with Ca. L. asiaticus (KJ944269). While two HLB isolates (CGB4, CGB5) shared nt identity of $96 \%$ and $92.7 \%$ with $C a$. $L$. asiaticus (EU265646, FJ821710) and Ca. L. asiaticus (EU130552) infecting citrus crop, respectively (Table 2). The nt identity of six (CGB1, CGB1a,
CGB2 CGB3 CGB4 and CGB5) HLB isolates is ranged from $63.6 \%$ to $73.5 \%$ with Ca. L. americanus isolates infecting citrus and periwinkle crop, $73.1 \%$ to $75.6 \%$ with Ca. L. africanus isolates infecting Clausena anisata, Teclea gerrardii wild citrus, $72.5 \%$ to $74 \%$ Ca. L. psyllaurous, Ca. L. solanacearum infecting tomato and potato, $70.0 \%$ to $71.6 \%$ with Ca. L. europaeus infecting Cytisus scoparius, respectively.

The phylogenetic tree based on comparison of $16 \mathrm{~S}$ rRNA gene sequence of HLB Coorg mandarin isolates characterized in this study with sequences of $C a$. $L$. 


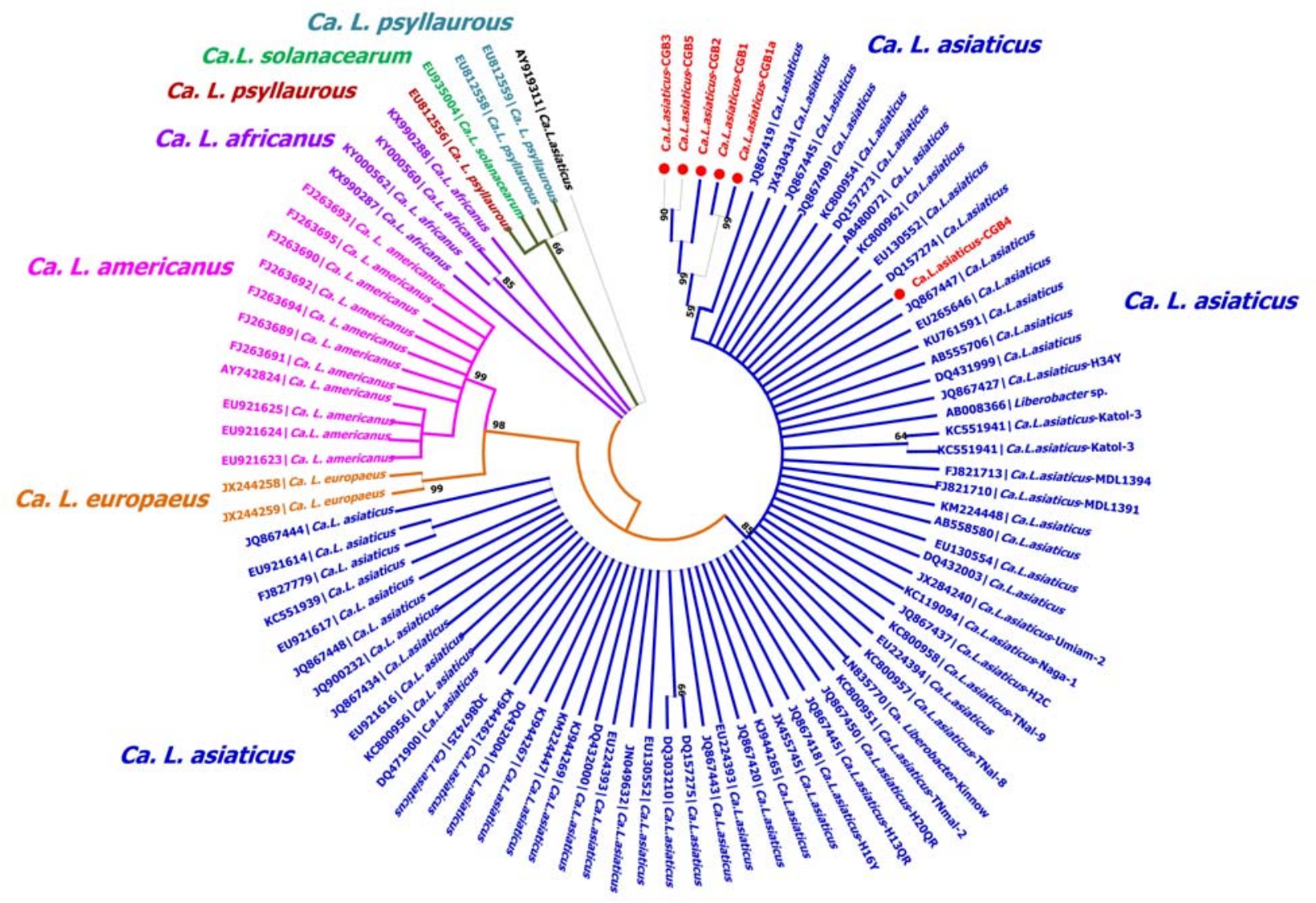

Ca. L. asiaticus

Fig. 6 Phylogenetic tree based on nt sequences of 16S rRNA gene of Ca. L. asiaticus with other Candidatus species using neighbor-joining algorithm.

Horizontal distances are proportional to sequence distances; vertical distances are arbitrary. The trees are unrooted. A bootstrap analysis with 1,000 replicates was performed and the bootstrap percent values more than 50 are numbered along branches.

asiaticus (65 isolates), Ca. L. americanus (11 isolates), Ca. L. africanus (four isolates), Ca. L. psyllaurous (three isolates), Ca. L. solanacearum (one isolate) identified previously on wild and cultivated species of citrus and solanaceous crops available in the database was shown in Fig. 6. The analysis showed that only one CGB4 isolate was closed cluster with Ca. $L$. asiaticus isolate infecting different citrus species and other five isolates (CGB1, CGB2, CGB3, CGB4 and CGB5) are form a separate cluster with all other Ca. $L$. asiaticus isolate. This indicates some variability existing in the Coorg mandarin isolates; this can be further addressed through amplification of other region of $16 \mathrm{~S}$ rRNA and restriction analysis. Further analysis also showed that Ca. L. asiaticus isolates from different countries grouped together while " $\mathrm{Ca}$. L. americanus", "Ca. L. africanus" and "Ca. L. psyllouraus" formed a separate and distinct group.

\section{Discussion}

The genus Phytophthora is a cosmopolitan oomycete belongs to the class Oomycetes, under the Phylum Heterokonta [19, 20]. The species of Phytophthora have a very wide host range distributed worldwide and cause economically important diseases in vegetables and fruit crops, ornamental plants and forest trees $[12,21]$. Citrus cultivation globally hampered due to infection of different Phytophthora spp., causing heavy yield losses and tree decline. Several Phytophthora species have been 
Table 5 The sequences of representative $P$. palmivora, $P$. nicotianae and citrus greening isolates are available in database under following accession number.

\begin{tabular}{ll}
\hline Phytophthora isolates & Accession number \\
\hline P. palmivora-FG7 & MF370562 \\
P. palmivora-FG8 & MF370563 \\
P. palmivora-FG9 & MF370564 \\
P. palmivora-FG10 & MF370565 \\
P. palmivora-FG11 & MF370567 \\
P. palmivora-FG12 & MF370568 \\
P. palmivora-FG30 & MF370569 \\
P. palmivora-FG34 & MF370570 \\
P. palmivora-FG38 & MF370571 \\
P. palmivora-FG41 & MF370572 \\
P. nicotianae-FG16 & MF370573 \\
P. nicotianae-FG17 & MF370574 \\
P. nicotianae-FG18 & MF370575 \\
P. nicotianae-FG20 & \\
\hline Citrus greening isolates & MF370576 \\
\hline Ca. L. asiaticus-CGB1 & MF370577 \\
Ca. L. asiaticus-CGB1a & MF370578 \\
Ca. L. asiaticus-CGB2 & MF370579 \\
Ca. L. asiaticus-CGB3 & MF370580 \\
Ca. L. asiaticus-CGB4 & MF370581 \\
Ca. L. asiaticus-CGB5 & \\
\hline
\end{tabular}

reported in different citrus growing regions of the world [12], among which P. nicotianae, P. palmivora and $P$. citrophthora are the most predominant species infecting citrus $[9,22]$ in various parts of India, i.e., Assam, Nagpur and Punjab [23-25].

The survey conducted in different location of Kodagu district of Karnataka (India) showed that most of the citrus plants in orchards were severely infected with Phytophthora, as well as HLB infection irrespective of age of the plants. The infected plants showed various degrees of disease symptom, such as yellowing, mottling, greening, root rot, trunk slit, wilting and gummosis, respectively. It was observed that budded plants express early susceptibility to both diseases then the plants raised from true seeds [26].

The Coorg mandarin samples (soil and infected feeder root) collected from different places of Kodagu districts revealed the distribution of $P$. palmivora in $60 \%$ of samples than $P$. nicotianae (41\%). This showed that $P$. palmivora was the most prevailing species infecting Coorg mandarin than $P$. nicotianae in Coorg region. Whereas, in other mandarin growing areas of India, P. nicotianae was reported to be more predominate species, which survived at higher temperature and infecting different citrus species rather than $P$. palmivora [9]. Similar results were found from various parts of world that $P$. palmivora and $P$. nicotianae are two pre-dominant species responsible for citrus decline [12, 22, 27-30]. This indicates that existing climatic condition and high rainfalls (average 1,010 $\mathrm{mm}$ ) in the Coorg region make Coorg mandarin more prone to $P$. palmivora infection.

It is difficult and inconvenient to identify some Phytophthora species based on morphological characteristics, as critical morphological features were variable and overlapping under different environmental conditions. Therefore, in the present study, $P$. palmivora and $P$. nicotianae were characterized through the amplification of ITS region that can rapidly distinguish between the two main species of Phytophthora involved in causing diseases 
in citrus [22]. The phylogenetic analysis based on ITS region revealed that $P$. palmivora and $P$. nicotianae isolates closely clustered with respective Phytopthora species infecting different citrus species in India and other different countries [9, 31]. Thus, the study clearly showed that Phytophthora isolates collected from 31 places of Kodagu district confirmed the presence of two Phytophthora species, namely $P$. palmivora and $P$. nicotianae, respectively.

Another important devastating disease of Coorg mandarin is citrus greening bacteria or HLB disease of Coorg mandarin. The presence of greening disease in different citrus species in India was recorded as early as 1965 , and the disease could be transmitted by budding through infected scion and psyllid (Diaphorina citri) as natural vector [32]. The association of greening bacterium in different citrus species was reported in India, excluding Coorg region [33]. The sampling survey conducted in the present study focused on major Coorg mandarin growing regions of Karnataka. During these surveys, several commercial Coorg mandarin fields displayed varied kind of disease symptoms, such as sparely foliated, extensive twig die-back, general yellowing, leaf mottling, mild to severe chlorosis, vein yellowing, pale green colour of young leaves. The preliminary analyses of 523 infected leaf samples of Coorg mandarin by PCR diagnostic tests indicated that the symptomatic plants collected from Coorg region in different commercial fields were infected with citrus greening bacterium. Similarly, technique was used in India to detect the greening bacterium different citrus species grown in different parts of India [6-8]. Greening disease on Coorg mandarin in Coorg region of Karnataka is more prevalent, but there was no information available on definite association and characterization of greening bacterium associated with it. Sequencing of representative six HLB isolates of 16S rRNA gene showed three isolates (CGB1, CGB1a, CGB3) is closely related with Ca. L. asiaticus (AB008366) isolate infecting citrus crop in Okinawa, one HLB isolate (CGB2) with Ca. L. asiaticus (KJ944269) infecting citrus in China. While two HLB isolates (CGB4, CGB5) are closely related to Ca. $L$. asiaticus (EU265646, FJ821710) and Ca. L. asiaticus (EU130552) infecting citrus in Florida, respectively. Therefore, the study clearly showed that Coorg mandarin decline in Coorg region is responsible for the infection of $P$. palmivora and $P$. nicotianae, as well as HLB bacterium (Ca. L. asiaticus), respectively.

\section{Conclusions}

Coorg mandarin is a famous ecotype of mandarin, grown in southern parts of India, particularly in Western Ghats as component of the multi-tier cropping system in coffee and pepper plantations for more than 150 years. The present study showed that two species Phytophthora and different strains of $\mathrm{Ca}$. L. asiaticus are causing the rot and greening disease on budded or true seeded Coorg mandarin plants. This may be due to several ground bed and container nurseries, many commercial citrus groves from respective regions were believed to be the hotspots for dissemination of these pathogens into new areas and hence need urgent quarantine attention. Using of disease free planting material along with effective integrated disease management (IDM) strategy is recommended to contain the diseases and to enhance the productivity of Coorg mandarin in Kodagu region.

\section{Acknowledgments}

The authors are grateful to the director of Indian Institute of Horticultural Research, Bangalore for providing research facilities and his keen interest in this study.

\section{References}

[1] Indian Horticultural Board. 2015 Indian Hortiuclture Database. http://nhb.gov.in/area-pro/NHB_Database_2015.

[2] Savita, G. S. V., and Nagpal, A. 2012. "Citrus Diseases Caused by Phytophthora Species." GERF Bulletin of Biosciences 3 (1): 18-27. 
[3] Timmer, L., Garnsey, S., and Broadbent, P. 2003. "Diseases of Citrus." In Diseases of Tropical Fruits Crops, edited by Ploetz, R. C. Wallingford, UK: CABI Publishing, CABI International, 163-95.

[4] Saunt, J. 1990. Citrus Varieties of the World. Norwich, Eng.: Sinclair International Ltd., 126.

[5] Zitko, S. E., and Timmer, L. W. 1994. "Competitive Parasitic Abilities of Phytophthora parasitica and $P$. palmivora on Fibrous Roots of Citrus." Phytopathology 84: 1000-4.

[6] Ahlawat, Y. S., Baranwal, V. K., and Mazumdar, S. 2003. "First Report of Citrus Greening Disease and Associated Bacterium 'Candidatus Liberibacter asiaticus' from Bhutan." Plant Dis. 87: 448.

[7] Baranwal, V. K., Mazumder, S., Singh, J., Suryanarayana, V., Ghosh, D. K., and Ahlawat, Y. S. 2004. "PCR Detection of Candidatus Liberibacter asiaticus, the Agent of Huanglongbin or Greening Disease in Citrus." Indian Phytopathol. 57 (2): 164-8.

[8] Das, A. K., Rao, C. N., and Singh, S. 2007. "Presence of Citrus Greening (Huanglongbing) Disease and Its Psyllid Vector in the North-Eastern Region of India Confirmed by PCR Technique." Curr. Sci. 92 (12): 1759-63.

[9] Das, A. K., Nerkar, S., Kumar, A., and Bawage, S. 2016. "Detection, Identification and Characterization of Phytophthora spp. Infecting Citrus in India." J. of Plant Pathol. 98 (1): 55-69.

[10] Garnier, M., Bové, J. M., Cronje, C. P. R., Sanders, G. M., Korsten, L., and Le Roux, H. F. 2000. "Presence of 'Candidatus Liberibacter africanus' in the Western Cape Province of South Africa." In Proceedings of 14th Conference IOCV, IOCV, Riverside, 369-72.

[11] Warcup, J. H. 1950. "The Soil-Plate Method for Isolation of Fungi from Soil." Nature 166: 117-8. doi:10.1038/166117b0.

[12] Erwin, D. C., and Ribeiro, O. K. 1996. Phytophthora Diseases Worldwide. St. Paul, USA: The American Phytopathological Society.

[13] Doyle, J. J., and Doyle, J. L. 1990. "Isolation of Plant DNA from Fresh Tissue." Focus 12: 13-5.

[14] White, T. M., Bruns, T., Lee, S., and Taylor, J. 1990. "Amplification and Direct Sequencing of Fungal Ribosomal RNA for Phylogenetics." In PCR Protocols: A Guide to Methods and Applications, edited by Innis, M. A., Gelfand, D. H., Sninsky, J. J., and White, T. J. San Diego, CA: Academic Press, 315-21.

[15] Jagoueix, S., Bove, J. M., and Garnier, M. 1996. "PCR Detection of the Two 'Candidatus' Liberobacter Species Associated with Greening Disease of Citrus." Molecular and Cellular Probes 10 (1): 43-50.

[16] Galtier, N., Gouy, M., Gautier, C. 1996. "SEAVIEW and PHYLO_WIN: Two Graphic Tools for Sequence
Alignment and Molecular Phylogeny." Comput. Applic. Biosci. 12 (6): 543-8.

[17] Hall, T. A. 1999. "BioEdit: A User-Friendly Biological Sequence Alignment Editor and Analysis Program for Windows 95/98/NT." Nucl. Acids 41: 95-8.

[18] Tamura, K., Peterson, D., Filipski, A., and Kumar, S. 2013. "MEGA6: Molecular Evolutionary Genetics Analysis Version 6.0.” Mol. Biol. Evol. 30 (12): 2725-9.

[19] Cavalier-Smith, T., and Chao, E. E. 2006. "Phylogeny and Megasystematics of Phagotrophic Heterokonts (Kingdom Chromista)." J. Mol. Evol. 62 (4): 388-420.

[20] Riisberg, I., Orr, R. J., Kluge, R., Shalchian-Tabrizi, K., Bowers, H. A., Patil, V., Edvardsen, B., and Jakobsen, K. S. 2009. "Seven Gene Phylogeny of Heterokonts." Protist 160 (2): 191-204. doi: 10.1016/j.protis.2008.11.004.

[21] Agrios, G. N. 2005. Plant Pathology, 5th ed.. New York, USA: Academic Press, 952. ISBN: 0120445654.

[22] Bowman, K. D., Albrecht, U., Graham, J. H., and Bright, D. B. 2007. "Detection of Phytophthora nicotianae and $P$. palmivora in Citrus Roots Using PCR-RFLP in Comparison with Other Methods." European Journal of Plant Pathology 119: 143-58.

[23] Chowdhary, S. 1951. "Gummosis of Citrus in Assam." Indian J. Agric. Sci. 16: 570-1.

[24] Bajwa, B. S. 1941. "Gummosis in Fruit Trees." Punjab Fruit J. 5: 889-91.

[25] Paracer, C. S., and Chahal, D. S. 1962. "Diseases of Root, Crown and Trunk of Citrus." Punjab Hort. J. 2: 92-5.

[26] Lele, V. C., and Kapoor, J. N. 1982. "Phytophthora on Citrus in Central and Peninsular India." Indian Phytopathology 35 (3): 407-10.

[27] Graham, J. H., Timmer, L. W., Drouillard, D. L., and Peever, T. L. 1998. "Characterisation of Phytophthora sp. Causing Outbreaks of Citrus Brown Rot in Florida." Phytopathology 88: 724-9.

[28] Graham, J. H., and Menge, J. A. 1999. "Root Diseases." In Citrus Health Management, edited by Timmer, L.W., and Duncan, L. W. St. Paul, MN, USA: APS Press, 126-35.

[29] Phung, M. H., Pongnak, W., Soytong, K., and Poeaim, S. 2015. "Identifications of Phytophthora spp. Causing Citrus Root Rots in Thailand." J. of Agric. Technol. 11 (8): 1897-910.

[30] Puglisi, I., De Patrizio, A., Schena, L., Jung, T., Evoli, M., and Pane, A. 2017. "Two Previously Unknown Phytophthora Species Associated with Brown Rot of Pomelo (Citrus grandis) Fruits in Vietnam." PLOS ONE 12 (2): e0172085. doi:10.1371/journal.pone.0172085.

[31] Nerkar, S. G., Bawage, S. S., Ashok, K., and Das, A. K. 2012. "Unigene-Derived Microsatellite Marker Based Variation Study of Phytophthora nicotianae Isolates Infecting Citrus.” Indian J. Microbiol. 52 (3): 489-91. 

Decline in Coorg Mandarin in India

[32] Capoor, S. P., Rao, D. G., and Viswanath, S. M. 1967. "Diaphorina citri Kuway, a Vector of the Greening Disease of Citrus in India." Indian J. Agric. Sci. 37: 572-6.

[33] Varma, A., Ahlawat, Y. S., Chakraborty, N. K., Garnier,
M., and Bove, J. M. 1993. "Detection of Greening BLO by Electron Microscopy, DNA Hybridization in Citrus Leaves with and without Mottle from Various Regions in India." In Proceedinds of 12th Conference of IOCV, Riverside, California, 280-5. 